\title{
Achieving Turbidity Robustness on Underwater Images Local Feature Detection
}

\section{Felipe Codevilla}

felipe.codevilla@furg.br

Joel De O. Gaya

joelfelipe@furg.br

Nelson Duarte Filho

dmtnldf@furg.br

Silvia S. C. Botelho

silviacb@furg.br
Center of Computational Sciences (C3)

Federal University of Rio Grande (FURG)

Rio Grande, Brazil
Methods to detect local features have been made to be invariant to many transformations. So far, the vast majority of feature detectors consider robustness just to over-land effects. However, when capturing pictures in underwater environments, there are media specific properties that can degrade the visual quality the captured images. Besides the shortening of information imaged, a corruption of information also happens. The main phenomena, the Backscattering, happens when reflected sources from outside the captured scene are scattered in a wide angle eventually reaching the image plane. This effect creates a characteristic veil on the image that reduces contrast and suppress fine structures on the image.

Little work has been made in order to study the robustness that the popular feature detectors have to underwater environment image conditions. In addition, applications benefited by finding descriptive feature points in underwater environments grows every year. They are essential for many applications like 3D reconstruction [4], visual odometry [6] and tracking [7]. Most of these applications rely on the best over-land feature detectors, without considering the water photometric properties. It is likely that some algorithms have a better behaviour than others when applied on images degraded by specific underwater conditions.

As stated before, underwater phenomena also create structural degradation. Further, it tends to eliminate all the finer scale structures, which is equivalent to the scale changing phenomena. Consequently, we propose the invariant points detected by some scale invariant detector can have also a good robustness to turbidity.

In this context, to evaluate feature detectors, we propose a new dataset called TURBID. This dataset is based on real underwater scenes photographs. The pictures are placed on the bottom of a tank filled with a milk-water solution and then are re-photographed with the degradation controlled by the amount of milk. The generated dataset for one of the printed photographs is show in Fig. 1. This dataset is an improvement in terms of visual diversity when compared to previous efforts [8] and is one of the main contributions of this work.

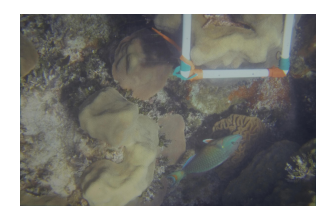

(a) Clean

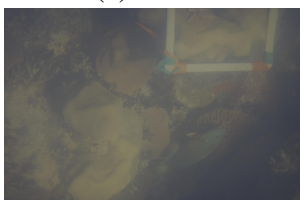

(a) $50 \mathrm{ml}$

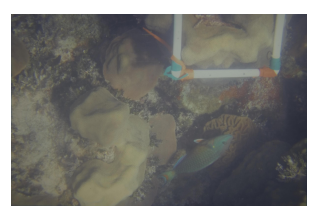

(b) $15 \mathrm{ml}$

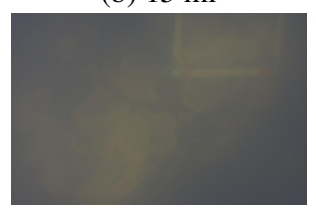

(b) $100 \mathrm{ml}$
Figure 1: The images captured over different levels of degradation due to turbidity controlled by milk addition. We photographed three different printed pictures. (a) Clean Image, no milk. (b) Low Turbidity with around $15 \mathrm{ml}$ of milk. (c) Medium Turbidity with around $50 \mathrm{ml}$ of milk. (d) High Turbidity with around $100 \mathrm{ml}$ of milk.

In order to analyze the robustness to turbidity we use the repeatability criteria on each obtained image. This criteria is proportional to the number of feature points found in the same spot given a error $\varepsilon$. The repeatability towards turbidity is calculated by the ratio of the number of points found in the clean image (Fig. 1(a)) and the number of points repeated in a turbid image, hence:

$$
R_{i}=\frac{N_{i}}{N_{0}}
$$

where $N_{0}$ is the number of features on the clear image, and $N_{i}$ is the number of features repeated on the image $T_{i}$.

For three different photos we computed the repeatability results. An example of this computation is show for Fig. 2.

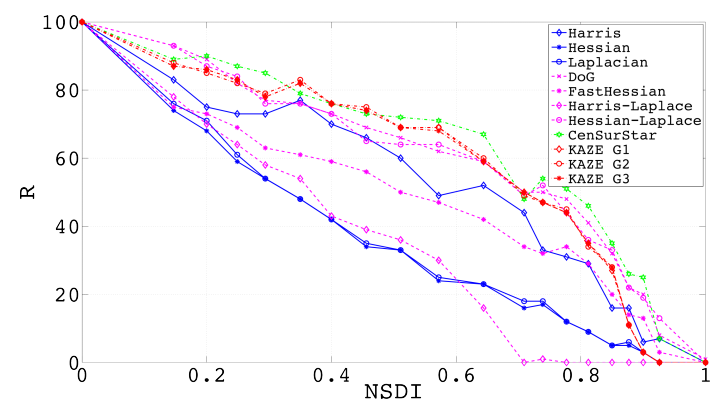

Figure 2: Results for $N=100$ and $\varepsilon=5$, showing the repeatability with respect to the ranging of the image degradation. For this case the CenSurStar [1] and KAZE [2] got the best results.

As we conjectured earlier, the Harris [9], the Hessian [5] and Laplacian approaches performed poorer than the scale invariant methods. Harris is generally used as very precise detector and is used in underwater tracking applications [7]. However, we show that, on the present scenario, the use of scale is useful also for precision.

Finally, we found that, finding scale invariant points is a useful way to find structural degradation robust points. We elected KAZE [2], Center Surround Extremas [1], Difference of Gaussians [10] and Fast Hessian[3] as good feature points detectors for underwater environments in all tested situations.

[1] Motilal Agrawal, Kurt Konolige, and Morten Rufus Blas. Censure: Center surround extremas for realtime feature detection and matching. In Computer Vision-ECCV 2008, pages 102-115. Springer, 2008.

[2] Pablo Fernández Alcantarilla, Adrien Bartoli, and Andrew J Davison. Kaze features. In Computer Vision-ECCV 2012, pages 214-227. Springer, 2012.

[3] Herbert Bay, Andreas Ess, Tinne Tuytelaars, and Luc Van Gool. Speeded-up robust features (surf). Computer vision and image understanding, 110, 2008.

[4] C. Beall, B.J. Lawrence, V. Ila, and F. Dellaert. 3d reconstruction of underwater structures. In IEEE/RSJ International Conference on Intelligent Robots and Systems (IROS), pages 4418-4423, 2010.

[5] P. R. Beaudet. Rotationally invariant image operators. In Proceedings of the 4th International Joint Conference on Pattern Recognition, pages 579-583, Kyoto, Japan, November 1978.

[6] S. S. C. Botelho, Paulo Drews-Jr, M. Figueiredo, C. Da Rocha, and G. L. Oliveira. Appearance-based odometry and mapping with feature descriptors for underwater robots. Journal of the Brazilian Computer Society, 15:47 54, 09 2009. ISSN 0104-6500.

[7] P. Corke, C. Detweiler, M. Dunbabin, M. Hamilton, D. Rus, and I. Vasilescu. Experiments with underwater robot localization and tracking. In Robotics and Automation, 2007 IEEE International Conference on, pages 4556-4561, 2007. doi: 10.1109/ROBOT.2007.364181.

[8] Rafael Garcia and Nuno Gracias. Detection of interest points in turbid underwater images. In OCEANS, 2011 IEEE-Spain, pages 1-9. IEEE, 2011.

[9] Chris Harris and Mike Stephens. A combined corner and edge detector. In Alvey vision conference, volume 15, page 50. Manchester, UK, 1988.

[10] David G Lowe. Distinctive image features from scale-invariant keypoints. International journal of computer vision, 60(2):91-110, 2004. 